\title{
Uniqueness of Meromorphic Functions of Differential Polynomials Sharing Two Values IM
}

\author{
Xinhua Shi \\ College of Science, Civil Aviation University of China, Tianjin, China \\ Email: xhshi2000@163.com
}

Received January 15, 2014; revised February 15, 2014; accepted February 21, 2014

Copyright (C) 2014 Xinhua Shi. This is an open access article distributed under the Creative Commons Attribution License, which permits unrestricted use, distribution, and reproduction in any medium, provided the original work is properly cited. In accordance of the Creative Commons Attribution License all Copyrights (C) 2014 are reserved for SCIRP and the owner of the intellectual property Xinhua Shi. All Copyright (C) 2014 are guarded by law and by SCIRP as a guardian.

\section{ABSTRACT}

In this paper, we shall study the uniqueness problems of meromorphic functions of differential polynomials sharing two values IM. Our results improve or generalize many previous results on value sharing of meromorphic functions.

\section{KEYWORDS}

Differential Polynomial; Uniqueness; Meromorphic Function; Shared Value

\section{Introduction}

Let $f(z)$ and $g(z)$ be two non-constant meromorphic functions defined in the open complex plane $\mathbf{C}$. Let $a \in \mathbf{C} \cup\{\infty\}$, we say that $f(z)$ and $g(z)$ share $a$ CM (counting multiplicities) if $f(z)-a, g(z)-a$ have the same zeros with the same multiplicities and we say that $f(z)$ and $g(z)$ share $a$ (ignoring multiplicities) if we do not consider the multiplicities. We denote by $T(r, f)$ the Nevanlinna characteristic function of the meromorphic function $f$ and by $S(r, f)$ any quantity satisfying $S(r, f)=o(T(r, f))$ as $r \rightarrow \infty$ possibly outside a set of finite linear measure. $N_{k}(r, f)$ denotes the truncated counting function bounded by $k$. Moreover, $G C D\left(n_{1}, n_{2}, \cdots, n_{k}\right)$ denotes the greatest common divisor of positive integers $n_{1}, n_{2}, \cdots, n_{k}$.

For the sake of simplicity, let $m$ be a nonnegative integer, $a_{0} \neq 0, a_{1}, \cdots, a_{m-1}, a_{m} \neq 0$ be complex constants. Define

$$
P(w)= \begin{cases}a_{m} w^{m}+a_{m-1} w^{m-1}+\cdots+a_{1} w+a_{0} & (m>0) \\ a_{0} & (m=0)\end{cases}
$$

In 1929, Nevanlinna [1] proved the following well-know result which is the so called Nevanlinna five values theorem.

Theorem A Let $f$ and $g$ be two non-constant meromorphic functions. If $f$ and $g$ share five distinct values $I M$, then $f \equiv g$.

Moreover, he got.

Theorem B Let $f$ and $g$ be two distinct non-constant meromorphic functions and $a_{j}(j=1,2,3,4)$ be four distinct values. If $f$ and $g$ share $a_{j}(j=1,2,3,4) C M$, then $f$ is a Mobius transformation of $g$.

In 1976, L. Rubel asked the following question:

Whether CM can be replaced by IM in the hypothesis of Theorem A with the same conclusion or not?

In 1979, G. G. Gundersen [2] gave a negative answer for this question by the following counterexample:

$$
f=\left(\mathrm{e}^{h}+1\right) /\left(\mathrm{e}^{h}-1\right)^{2}, \quad g=\left(\mathrm{e}^{h}+1\right)^{2} /\left[8\left(\mathrm{e}^{h}-1\right)\right],
$$


where $h$ is a non-constant entire function. It is easy to verify that $f$ and $g$ share the four values $0,1, \infty,-1 / 8$, where none of the four values are shared CM, and $f$ is not a Mobius transformation of $g$.

On the other hand, G. G. Gundersen [3] proved the following result which is an improvement of Theorem B. Theorem C. If two distinct non-constant meromorphic functions share two values CM and share two other values IM, then the functions share all four values CM (hence the conclusions of Theorem B hold).

In this paper, we shall show that similar conclusions hold for certain types of differential polynomials when they share two values IM.

Theorem 1.1 Let $f$ and $g$ be two non-constant meromorphic functions, $n>0, k>0$, and $m \geq 0$ be three integers with $n>4 m+9 k+10$ and $P(w)$ be defined as in (1.1). If $\left[f^{n} P(f)\right]^{(k)}$ and $\left[g^{n} P(g)\right]^{(k)}$ share 1 and $\infty$ IM, then

1) when $m>0, \quad f^{n} P(f) \equiv g^{n} P(g)$;

2) when $m=0$, one of the following two cases holds:

3) $f \equiv \operatorname{tg}$ for a constant $t$ such that $t^{n}=1$,

4) $f(z)=c_{1} / \sqrt[n]{a_{0}} \mathrm{e}^{c z}, g(z)=c_{2} / \sqrt[n]{a_{0}} \mathrm{e}^{-c z}$, where $c_{1}, c_{2}$ and $c$ are three constants satisfying $(-1)^{k}\left(c_{1} c_{2}\right)^{n}(n c)^{2 k}=1$.

Remark 1.1 “ $\left[f^{n} P(f)\right]^{(k)}$ and $\left[g^{n} P(g)\right]^{(k)}$ share $\infty$ IM" $\Leftrightarrow f$ and $g$ share $\infty$ IM". Moreover, from $f^{n} P(f) \equiv g^{n} P(g)$, one cannot get $f \equiv t g$ for some constant $t$. For example, let $P(f)=f-1$, $P(g)=g-1$, then $f=\left(1-h^{n}\right) h /\left(1-h^{n+1}\right), g=\left(1-h^{n}\right) /\left(1-h^{n+1}\right)$, where $h$ is a non-constant meromorphic function. Obviously, $f \equiv \equiv$ tg for some canstant $t$ but $f^{n}(f-1) \equiv g^{n}(g-1)$.

Now we give some corollaries of Theorem 1.1. Corollary 1.2 and Corollary 1.3 improve Theorems D and E, respectively.

Corollary 1.2 Let $f$ and $g$ be two non-constant meromorphic functions, and let $n, k$ be two positive integers with $n>9 k+10$. If $\left[f^{n}\right]^{(k)}$ and $\left[g^{n}\right]^{(k)}$ share $1 \mathrm{IM}, f$ and $g$ share $\infty$ IM, then either $f(z)=c_{1} \mathrm{e}^{c z}, g(z)=c_{2} \mathrm{e}^{-c z}$, where $c_{1}, c_{2}$ and $c$ are three constants satisfying $(-1)^{k}\left(c_{1} c_{2}\right)^{n}(n c)^{2 k}=1$, or $f \equiv \operatorname{tg}$ for a constant $t$ such that $t^{n}=1$.

Corollary 1.3 Let $f$ and $g$ be two non-constant meromorphic functions satisfying $\Theta(\infty, f)>2 / n$, and let $n, k$ be two positive integers with $n>9 k+14$. If $\left[f^{n}(f-1)\right]^{(k)}$ and $\left[g^{n}(g-1)\right]^{(k)}$ share $1 \mathrm{IM}, f$ and $g$ share $\infty$ IM, then $f \equiv g$.

Corollary 1.4 Let $f$ and $g$ be two non-constant meromorphic functions, and let $n, k, m(\geq 2)$ be two positive integers with $n>4 m+9 k+10, a$ be a nonzero constant. If $\left[f^{n}\left(f^{m}+a\right)\right]^{(k)}$ and $\left[g^{n}\left(g^{m}+a\right)\right]^{(k)}$ share $1 \mathrm{IM}, f$ and $g$ share $\infty \mathrm{IM}$, then $f \equiv t g$ for some constant $t$ such that $t^{d}=1$, where $d=G C D(n+m, n)$.

Theorem 1.1 generalizes the following result that was obtained by Zhang, Chen and Lin [4].

Theorem D Let $f$ and $g$ be two non-constant entire functions. Let $n, k$, and $m$ be three positive integers with $n \geq 3 m+2 k+5$ and let $P(w)=a_{m} w^{m}+a_{m-1} w^{m-1}+\cdots+a_{1} w+a_{0}$ or $P(w) \equiv c_{0}$, where $a_{0} \neq 0, a_{1}, \cdots, a_{m-1}, a_{m} \neq 0, c_{0} \neq 0$ are complex constants. If $\left[f^{n} P(f)\right]^{(k)}$ and $\left[g^{n} P(g)\right]^{(k)}$ share $1 \mathrm{CM}$, then

1) when $P(w)=a_{m} w^{m}+a_{m-1} w^{m-1}+\cdots+a_{1} w+a_{0}$, either $f \equiv t g$ for a constant $t$ such that $t^{d}=1$, where $d=G C D(n+m, \cdots, n+m-i, \cdots, n), \quad a_{m-i} \neq 0$ for some $i=0,1, \cdots, m$, or $f$ and $g$ satisfy the algebraic equation $R(f, g) \equiv 0$,

where $R\left(\omega_{1}, \omega_{2}\right)=\omega_{1}^{n}\left(a_{m} \omega_{1}^{m}+a_{m-1} \omega_{1}^{m-1}+\cdots+a_{0}\right)-\omega_{2}^{n}\left(a_{m} \omega_{2}^{m}+a_{m-1} \omega_{2}^{m-1}+\cdots+a_{0}\right)$;

2) when $P(w) \equiv c_{0}$, either $f(z)=c_{1} / \sqrt[n]{c_{0}} \mathrm{e}^{c z}, g(z)=c_{2} / \sqrt[n]{c_{0}} \mathrm{e}^{-c z}$, where $c_{1}, c_{2}$ and $c$ are three constants 
satisfying $(-1)^{k}\left(c_{1} c_{2}\right)^{n}(n c)^{2 k}=1$, or $f \equiv t g$ for a constant $t$ such that $t^{n}=1$.

Corollaries 1.2-1.4 greatly improve the following result that was obtained by Liu [5] by reducing the lower bound of $n$. Moreover, the proofs of Corollaries $1.2-1.4$ fill some gaps appeared in the proof of Theorem E.

Theorem E Let $f$ and $g$ be two non-constant meromorphic functions, and let $n, k$, and $m$ be three positive integers with $n>6 m^{*}+9 k+14$, and $\lambda, \mu$ be two constants such that $|\lambda|+|\mu| \neq 0$. If

$\left[f^{n}\left(\mu f^{m}+\lambda\right)\right]^{(k)}$ and $\left[g^{n}\left(\mu g^{m}+\lambda\right)\right]^{(k)}$ share $1 \mathrm{IM}, f$ and $g$ share $\infty$ IM, then $\backslash$

1) when $\lambda \mu \neq 0$, If $m \geq 2$ and $\delta(\infty, f)>3 /(m+n)$, then $f \equiv g$.

If $m=1$ and $\delta(\infty, f)>3 /(n+1)$, then $f \equiv g$;

2) when $\lambda \mu=0$, if $f \neq \infty$ and $g \neq \infty$, then either $f \equiv t g$, where $t$ is a constant satisfying $t^{n+m^{*}}=1$, or $f(z)=c_{1} \mathrm{e}^{c z}, g(z)=c_{2} \mathrm{e}^{-c z}$, where $c_{1}, c_{2}$ and $c$ are three constants satisfying

$(-1)^{k} \lambda^{2}\left(c_{1} c_{2}\right)^{n+m^{*}}\left(\left(n+m^{*}\right) c\right)^{2 k}=1$ or $(-1)^{k} \mu^{2}\left(c_{1} c_{2}\right)^{n+m^{*}}\left(\left(n+m^{*}\right) c\right)^{2 k}=1$ Here, $m^{*}:=\chi_{\mu} m$, where $\chi_{\mu}=0$ if $\mu=0, \chi_{\mu}=1$ if $\mu \neq 0$.

\section{Preliminary Lemmas}

Let

$$
\begin{gathered}
H=F^{\prime \prime} / F^{\prime}-2 F^{\prime} /(F-1)-G^{\prime \prime} / G^{\prime}+2 G^{\prime} /(G-1), \\
V=F^{\prime} /(F-1)-F^{\prime} / F-G^{\prime} /(G-1)+G^{\prime} / G,
\end{gathered}
$$

where $F$ and $G$ are meromorphic functions.

Lemma 2.1 [6] Let $f$ be a non-constant meromorphic function and let $a_{0}(z), a_{1}(z), \cdots, a_{n}(z)(\equiv 0)$ be small functions with respect to $f$. Then

$$
T\left(r, a_{n} f^{n}+a_{n-1} f^{n-1}+\cdots+a_{0}\right)=n T(r, f)+S(r, f) .
$$

Lemma 2.2 [7] Let $f(z)$ be a non-constant meromorphic function, $s, k$ be two positive integers. Then

$$
\begin{gathered}
N_{s}\left(r, 1 / f^{(k)}\right) \leq T\left(r, f^{(k)}\right)-T(r, f)+N_{s+k}(r, 1 / f)+S(r, f), \\
N_{s}\left(r, 1 / f^{(k)}\right) \leq k \bar{N}(r, f)+N_{s+k}(r, 1 / f)+S(r, f) .
\end{gathered}
$$

Lemma 2.3 [8-10] Let $f(z)$ be a non-constant meromorphic function, and let $k$ be a positive integer. Suppose that $f^{(k)} \neq 0$, then

$$
N\left(r, 1 / f^{(k)}\right) \leq N(r, 1 / f)+k \bar{N}(r, f)+S(r, f) .
$$

By using the similar method to Banerjee [11, Lemma 2.14], we can prove the following Lemma.

Lemma 2.4 Let $F, G$ and $H$ be defined as in (2.1). If $F$ and $G$ share $1 \mathrm{CM}$ and $\infty \mathrm{IM}$, and $H \neq 0$, then $F \neq \equiv G$, and

$$
T(r, F) \leq N_{2}(r, 1 / F)+N_{2}(r, 1 / G)+2 \bar{N}(r, 1 / F)+\bar{N}(r, 1 / G)+6 \bar{N}(r, F)+S(r, F)+S(r, G),
$$

the same inequality holding for $T(r, G)$.

Lemma 2.5 [12] Let $F, G$ and $V$ be defined as in (2.2). If $F$ and $G$ share $\infty$ IM, and $V \equiv 0$, then $F \equiv G$.

Lemma 2.6 [13] If $F$ and $G$ share 1 IM, then

$$
\bar{N}_{L}(r, 1 /(F-1)) \leq \bar{N}(r, 1 / F)+\bar{N}(r, F)+S(r, F)+S(r, G) .
$$

Lemma 2.7 Let $f, g$ be two non-constant meromorphic functions, $V$ be defined as in (2.2), where $F=\left[f^{n} P(f)\right]^{(k)}, G=\left[f^{n} P(g)\right]^{(k)}, P(w)$ is defined as in (1.1), $n>0, k>0$ and $m \geq 0$ are three integers. If $V \neq 0, F$ and $G$ share $1 \mathrm{CM}$ and $\infty$ IM, then 


$$
(n+m+k-3) \bar{N}(r, f)=(n+m+k-3) \bar{N}(r, g) \leq 2 \bar{N}(r, 1 / F)+2 \bar{N}(r, 1 / G)+S(r, f)+S(r, g) .
$$

Proof Since $V \equiv 0, f$ and $g$ share $\infty$ IM, suppose that $z_{0}$ is a pole of $f$ with multiplicity $p$, a pole of $g$ with multiplicity $q$, then $z_{0}$ is a pole of $F$ with multiplicity $(n+m) p+k$, a pole of $G$ with multiplicity $(n+m) q+k$, thus $z_{0}$ is a zero of $F^{\prime} /(F-1)-F^{\prime} / F=F^{\prime} /[F(F-1)]$ with multiplicity $(n+m) p+k-1(\geq n+m+k-1)$, and $z_{0}$ is a zero of $G^{\prime} /(G-1)-G^{\prime} / G=G^{\prime} /[G(G-1)]$ with multiplicity $(n+m) q+k-1(\geq n+m+k-1)$, hence $z_{0}$ is a zero of $V$ with multiplicity at least $n+m+k-1$. So

$$
(n+m+k-1) \bar{N}(r, f)=(n+m+k-1) \bar{N}(r, g) \leq N(r, 1 / V) .
$$

By the logarithmic derivative lemma, we have $m(r, V)=S(r, f)+S(r, g)$. Note that $F$ and $G$ share 1 IM, by Lemma 2.6, so we have

$$
\begin{aligned}
N(r, 1 / V) & \leq T(r, V)=m(r, V)+N(r, V) \\
& \leq \bar{N}(r, 1 / F)+\bar{N}(r, 1 / G)+\bar{N}_{L}\left(r, \frac{1}{F-1}\right)+\bar{N}_{L}\left(r, \frac{1}{G-1}\right)+S(r, f)+S(r, g) \\
& \leq 2 \bar{N}(r, 1 / F)+2 \bar{N}(r, 1 / G)+2 \bar{N}(r, f)+S(r, f)+S(r, g) .
\end{aligned}
$$

From (2.4) and (2.5) we get (2.3). This proves Lemma 2.7.

Lemma 2.8 [14] Let $f$ and $g$ be two non-constant meromorphic functions, and $k, n>2 k+1$ be two positive integers. If $\left[f^{n}\right]^{(k)}=\left[g^{n}\right]^{(k)}$, then $f \equiv \operatorname{tg}$ for a constant $t$ such that $t^{n}=1$.

By the same reason as in Lemma 5 of [8], we obtain the following lemma.

Lemma 2.9 Let $f$ and $g$ be two non-constant meromorphic functions. Let $P(w)$ be defined as in (1.1), and $n>0, k>0$, and $m \geq 0$ be three integers with $n>2 k+m+1$. If $\left[f^{n} P(f)\right]^{(k)}=\left[g^{n} P(g)\right]^{(k)}$, then $f^{n} P(f)=g^{n} P(g)$.

Lemma 2.10 [15] Let $f$ and $g$ be non-constant meromorphic functions, $n, k$ be two positive integers with $n>k+2$, and let $P(w)$ be defined as in (1.1), $a(z)(\equiv 0, \infty)$ be a small function with respect to $f$ with finitely many zeros and poles. If $\left[f^{n} P(f)\right]^{(k)}\left[g^{n} P(g)\right]^{(k)}=a^{2}, f$ and $g$ share $\infty$ IM, then $P(w)$ is reduced to a nonzero monomial.

Use the proof of Theorem 3 in [15] and we obtain.

Lemma 2.11 Let $f$ and $g$ be non-constant meromorphic functions, $n, k$ be two positive integers with $n>k$. If $\left[f^{n}\right]^{(k)}\left[g^{n}\right]^{(k)}=1, f$ and $g$ share $\infty \mathrm{IM}$, then $f(z)=c_{1} \mathrm{e}^{c z}, g(z)=c_{2} \mathrm{e}^{-c z}$, where $c_{1}, c_{2}$ and $c$ are three constants satisfying $(-1)^{k}\left(c_{1} c_{2}\right)^{n}(n c)^{2 k}=1$.

Lemma 2.12 [16] Let $s>0$ and $t$ are relatively prime integers, and let $c$ be a complex number such that $c^{s}=1$. Then there exists one and only one common zero of $w^{s}-1$ and $w^{t}-c$.

\section{Proof of Theorem 1.1}

Let $F=\left[f^{n} P(f)\right]^{(k)}, G=\left[g^{n} P(g)\right]^{(k)}, F_{1}=f^{n} P(f), G_{1}=g^{n} P(g)$, then $F$ and $G$ share 1 IM and $\infty$

IM. Suppose that $H \not \equiv 0$, then $F \neq \equiv$, and $V \not 0$.

Case 1. $m>0$. By Lemma 2.4 we have

$$
T(r, F) \leq N_{2}(r, 1 / F)+N_{2}(r, 1 / G)+2 \bar{N}(r, 1 / F)+\bar{N}(r, 1 / G)+6 \bar{N}(r, F)+S(r, F)+S(r, G),
$$

By Lemma 2.2 with $s=2$, we obtain

$$
T\left(r, F_{1}\right) \leq T(r, F)-N_{2}(r, 1 / F)+N_{k+2}\left(r, 1 / F_{1}\right)+S(r, F),
$$


and

$$
N_{2}(r, 1 / G) \leq N_{k+2}\left(r, 1 / G_{1}\right)+k \bar{N}(r, G)+S(r, G) .
$$

Combining (3.1) - (3.3) gives

$$
\begin{aligned}
T\left(r, F_{1}\right) \leq & N_{k+2}\left(r, 1 / F_{1}\right)+N_{k+2}\left(r, 1 / G_{1}\right)+(4 k+6) \bar{N}(r, f) \\
& +2 N_{k+1}\left(r, 1 / F_{1}\right)+N_{k+1}\left(r, 1 / G_{1}\right)+S(r, f)+S(r, g) \\
\leq & (3 k+4) \bar{N}(r, 1 / f)+3 N(r, 1 / P(f))+(2 k+3) \bar{N}(r, 1 / g) \\
& +2 N(r, 1 / P(g))+(4 k+6) \bar{N}(r, f)+S(r, f)+S(r, g) .
\end{aligned}
$$

It follows from Lemma 2.1 and the above inequality that

$$
\begin{aligned}
(n+m) T(r, f) \leq & (3 k+4) \bar{N}(r, 1 / f)+3 N(r, 1 / P(f))+(2 k+3) \bar{N}(r, 1 / g) \\
& +2 N(r, 1 / P(g))+(4 k+6) \bar{N}(r, f)+S(r, f)+S(r, g) \\
\leq & (3 k+3 m+4) T(r, f)+(2 k+2 m+3) T(r, g) \\
& +(4 k+6) \bar{N}(r, f)+S(r, f)+S(r, g) .
\end{aligned}
$$

Similarly we have

$$
\begin{aligned}
(n+m) T(r, g) \leq & (3 k+3 m+4) T(r, g)+(2 k+2 m+3) T(r, f) \\
& +(4 k+6) \bar{N}(r, g)+S(r, f)+S(r, g) .
\end{aligned}
$$

Note that. $\bar{N}(r, f)=\bar{N}(r, g)$. From (3.4) and (3.5) we deduce that

$$
(n-5 k-4 m-7)(T(r, f)+T(r, g)) \leq 2(4 k+6) \bar{N}(r, f)+S(r, f)+S(r, g) \text {. }
$$

Note that $V \neq 0$ and we get (2.3). By Lemma 2.2 with $s=1$, we obtain

$$
\begin{aligned}
N_{1}(r, 1 / F) & =\bar{N}(r, 1 / F) \leq N_{k+1}\left(r, 1 / F_{1}\right)+k \bar{N}(r, f)+S(r, f) \\
& \leq(k+1) \bar{N}(r, 1 / f)+N(r, 1 / P(f))+k \bar{N}(r, f)+S(r, f),
\end{aligned}
$$

and

$$
\begin{aligned}
N_{1}(r, 1 / G) & =\bar{N}(r, 1 / G) \leq N_{k+1}\left(r, 1 / G_{1}\right)+k \bar{N}(r, g)+S(r, g) \\
& \leq(k+1) \bar{N}(r, 1 / g)+N(r, 1 / P(g))+k \bar{N}(r, g)+S(r, g) .
\end{aligned}
$$

From (2.3), (3.7) and (3.8) we get

$$
(n+m-3 k-3) \bar{N}(r, f) \leq 2(k+m+1)(T(r, f)+T(r, g))+S(r, f)+S(r, g) .
$$

Combining (3.6) - (3.9) gives

$$
[(n-5 k-4 m-7)(n+m-3 k-3)-4(4 k+6)(k+m+1)](T(r, f)+T(r, g)) \leq S(r, f)+S(r, g),
$$

which is a contradiction since $n>4 m+9 k+10$. Thus $H \equiv 0$. Similar to the proof of [17, Lemma 3], we obtain

1) $\left[f^{n} P(f)\right]^{(k)}\left[g^{n} P(g)\right]^{(k)}=1$, or

2) $\left[f^{n} P(f)\right]^{(k)} \equiv\left[g^{n} P(g)\right]^{(k)}$.

By Lemma 2.10, the case of 1) is impossible. By Lemma 2.9, we get $f^{n} P(f) \equiv g^{n} P(g)$ from 2).

Case 2. $m=0$. Similar to the proof of Case 1 , we get

$$
[(n-5 k-7)(n-3 k-3)-4(4 k+6)(k+1)](T(r, f)+T(r, g)) \leq S(r, f)+S(r, g),
$$

which is a contradiction since $n>9 k+10$. Thus $H \equiv 0$. and we have

3) $\left[f^{n}\right]^{(k)}\left[g^{n}\right]^{(k)}=1$, or 
4) $\left[f^{n}\right]^{(k)} \equiv\left[g^{n}\right]^{(k)}$.

For 3), by Lemma 2.11, we get $f(z)=c_{1} / \sqrt[n]{a_{0}} \mathrm{e}^{c z}, g(z)=c_{2} / \sqrt[n]{a_{0}} \mathrm{e}^{-c z}$, where $c_{1}, c_{2}$ and $c$ are three constants satisfying $(-1)^{k}\left(c_{1} c_{2}\right)^{n}(n c)^{2 k}=1$.

For 4), By Lemma 2.8, we get $f \equiv \operatorname{tg}$ for a constant $t$ such that $t^{n}=1$. This completes the proof of Theorem 1.1.

\section{Proof of Corollaries 1.2 - 1.4}

The proof of Corollary 1.2 is the same to the proof of Case 2 of Theorem 1.1 , we only need to let $a_{0}=1$. Thus we omit the proof here.

Now we prove Corollary 1.3, Let $m=1$, similar to (3.10), we get

$$
[(n-5 k-11)(n-3 k-2)-4(4 k+6)(k+2)](T(r, f)+T(r, g)) \leq S(r, f)+S(r, g),
$$

which is a contradiction since $n>9 k+14$. Thus $H \equiv 0$ and we have

1) $\left[f^{n}(f-1)\right]^{(k)}\left[g^{n}(g-1)\right]^{(k)}=1$, or

2) $\left[f^{n}(f-1)\right]^{(k)} \equiv\left[g^{n}(g-1)\right]^{(k)}$.

By Lemma 2.10, the case of (i) is impossible. By Lemma 2.9, we get $f^{n}(f-1) \equiv g^{n}(g-1)$ from 2).

Similar to the proof of Theorem 2 in [14], we get $f \equiv g$. This proves Corollary 1.3.

Next we prove Corollary 1.4.

According to the proof of Case 1 in Theorem 1.1, we have

1) $\left[f^{n}\left(f^{m}-1\right)\right]^{(k)}\left[g^{n}\left(g^{m}-1\right)\right]^{(k)}=1$, or

2) $\left[f^{n}\left(f^{m}-1\right)\right]^{(k)} \equiv\left[g^{n}\left(g^{m}-1\right)\right]^{(k)}$.

By Lemma 2.10, the case of 1$)$ is impossible. By Lemma 2.9, we get $f^{n}\left(f^{m}-1\right) \equiv g^{n}\left(g^{m}-1\right)$ from 2).

Let $h=f / g$. If $h$ is not a constant, then substitute $f=g h$ into $f^{n}\left(f^{m}-1\right) \equiv g^{n}\left(g^{m}-1\right)$ and we get

$$
g^{m}=\frac{1-h^{n}}{1-h^{n+m}}=\frac{\left(h-a_{1}\right) \cdots\left(h-a_{n}\right)}{\left(h-b_{1}\right) \cdots\left(h-b_{n+m}\right)},
$$

where $a_{j}(j=1,2, \cdots, n)$ are distinct roots of the algebraic equation $l^{n}=1, b_{j}(j=1,2, \cdots, n+m)$ are distinct roots of the algebraic equation $l^{n+m}=1$.

Suppose that $d=G C D(n+m, n)$, then $n+m=s d, n=t d$, where $s, t$ are co-prime integers and $s>t$, thus $m=(s-t) d$, which implies $d \leq m$. By Lemma 2.12, there exists one and only one common zero of $w^{s}-1$ and $w^{t}-1$, namely $w=1$. Therefore, there exists at least $n$ of $b_{j}(j=1,2, \cdots, n+m)$ different from $a_{j}(j=1,2, \cdots, n)$. Suppose that $b_{j}(j=1,2, \cdots, n)$ are different from $a_{j}(j=1,2, \cdots, n)$, then all zeros of $h-b_{j}(j=1,2, \cdots, n)$ have order of at least m. Applying the second fundamental theorem to $h$ gives

$$
(n-2) T(r, h) \leq \sum_{j=1}^{n} \bar{N}\left(r, \frac{1}{h-b_{j}}\right)+S(r, h) \leq \frac{1}{2} \sum_{j=1}^{n} N\left(r, \frac{1}{h-b_{j}}\right)+S(r, h) \leq \frac{n+1}{2} T(r, h)+S(r, h) .
$$

Note that $n>4 m+9 k+10$ and we get a contradiction. Thus $h$ is a constant. From (4.2) we have $h^{n+m}=1$ and $h^{n}=1$, thus $f \equiv t g$ for some constant $t$ such that $t^{d}=1$, where $d=G C D(n+m, n)$. This proves Corollary 1.4 .

\section{Open Problem}

For further study, we pose the following. Problem: What form of $f^{n} P(f) \equiv g^{n} P(g)$ implies $f \equiv t g$ for some constant $t$ ? 


\section{Acknowledgements}

The author would like to thank the referee for his valuable suggestions.

\section{REFERENCES}

[1] R. Nevanliana, "Le Theoreme de Picard-Borel at la Theorie des Fonctions Meromorph,” Gauthier-Villars, Paries, 1929.

[2] G. G. Gundersen, "Meromorphic Functions That Share Three or Four Values,” Journal of the London Mathematical Society, Vol. 20, No. 2, 1979, pp. 457-466. http://dx.doi.org/10.1112/jlms/s2-20.3.457

[3] G. G. Gundersen, “Meromorphic Functions That Share Four Values,” Transactions of the American Mathematical Society, Vol. 277, No. 2, 1983, pp. 545-567.

[4] X. Y. Zhang, J. F. Chen and W. C. Lin, “Entire or Meromorphic Functions Sharing One Value,” Computers \& Mathematics with Applications, Vol. 56, No. 7, 2008, pp. 1876-1883. http://dx.doi.org/10.1016/j.camwa.2008.04.008

[5] L. P. Liu, “Uniqueness of Meromorphic Functions and Differential Polynomials," Computers \& Mathematics with Applications, Vol. 56, No. 12, 2008, pp. 3236-3245. http://dx.doi.org/10.1016/j.camwa.2008.09.020

[6] C. C. Yang, “On Deficiencies of Differential Polynomials II,” Mathematische Zeitschrift, Vol. 125, No. 2, 1972 , pp. $107-112$. http://dx.doi.org/10.1007/BF01110921

[7] W. C. Lin and H. X. Yi, "Uniqueness Theorems for Meromorphic Functions," Indian Journal of Pure and Applied Mathematics, Vol. 35, No. 2, 2004, pp. 121-132.

[8] W. K. Hayman, “Meromorphic Functions,” Clarendon Press, Oxford, 1964.

[9] C. C. Yang and H. X. Yi, “Uniqueness Theory of Meromorphic Functions,” Kluwer Academic Publication, Dordrecht, 2003. http://dx.doi.org/10.1007/978-94-017-3626-8

[10] L. Yang, "Value Distribution Theory,” Springer-Verlag, Berlin, 1993.

[11] A. Banerjee, "Meromorphic Functions Sharing One Value,” International Journal of Mathematics and Mathematical Sciences, Vol. 2005, No. 22, 2005, pp. 3587-3598. http://dx.doi.org/10.1155/IJMMS.2005.3587

[12] H. X. Yi, “Meromorphic Functions That Share Three Sets,” Kodai Mathematical Journal, Vol. 20, No. 1, 1997, pp. $22-32$. http://dx.doi.org/10.2996/kmj/1138043717

[13] H. X. Yi, “Meromorphic Functions That Share One or Two Values. II,” Kodai Mathematical Journal, Vol. 22, No. 2, 1999, pp. 264-272. http://dx.doi.org/10.2996/kmj/1138044046

[14] J. F. Xu, F. Lv and H. X. Yi, "Fixed-Points and Uniqueness of Meromorphic Functions,” Computers \& Mathematics with Applications, Vol. 59, No. 1, 2010, pp. 9-17. http://dx.doi.org/10.1016/j.camwa.2009.07.024

[15] X. B. Zhang and J. F. Xu, "Uniqueness of Meromorphic Functions Sharing a Small Function and Its Applications," Computers \& Mathematics with Applications, Vol. 61, No. 3, 2011, pp. 722-730. http://dx.doi.org/10.1016/j.camwa.2010.12.022

[16] Q. C. Zhang, "Meromorphic Functions Sharing Three Values,” Indian Journal of Pure and Applied Mathematics, Vol. 30, No. 7, 1999, pp. 667-682.

[17] C. C. Yang and X. H. Hua, "Uniqueness and Value-Sharing of Meromorphic Functions," Annales Academice Scientiarum Fennicce Mathematica, Vol. 22, No. 2, 1997, pp. 395-406. 